\title{
Deer Antlers - A Model of Mammalian Appendage Regeneration: An Extensive Review
}

\author{
Uwe Kierdorf Horst Kierdorf \\ Department of Biology, University of Hildesheim, Hildesheim, Germany
}

\section{Key Words}

Antlers · Epimorphic regeneration • Periosteum •

Regenerative medicine $\cdot$ Stem cells

\begin{abstract}
Background: Compared with other vertebrate taxa, mammals possess a very limited capacity for appendage regeneration. The antlers of deer are an exception in that they are periodically lost and fully regenerated throughout the life of an individual. Objective: In this paper we compare certain aspects of antler regeneration with regenerative processes in other vertebrates. Methods: Review of the literature. Results: Recent studies suggest that antler regeneration is a stem cell-based process and that these stem cells are located in the pedicle periosteum. There is evidence that signaling pathways known to operate during appendage regeneration in other vertebrates are also activated during antler regeneration. There are, however, also differences between antlers and other systems of epimorphic regeneration. Thus, contrary to amphibian limb regeneration, signaling from the wound epidermis appears not to be of crucial importance for antler regeneration. Healing of the casting wound typically involves no or only minor scarring, making antlers interesting subjects for researchers attempting to reduce scar formation during wound healing in humans. The fact that despite their enormous growth rate the antlers of intact and castrated deer appear to be resistant to malignant transformation furthermore offers research opportunities for cancer biology. Conclusions: Studying antler renewal as an exam-
\end{abstract}

ple of mammalian appendage regeneration may provide crucial information for regenerative medicine to achieve its ultimate goal of stimulating limb regeneration in humans. A deeper understanding of the developmental mechanisms involved in antler renewal can also be useful for controlling induced regeneration processes in mammals.

Copyright $\odot 2010$ S. Karger AG, Basel

\section{Introduction}

The ability for appendage regeneration varies considerably among vertebrates, with urodele amphibians possessing the greatest regenerative capacity. Thus, newts or salamanders can fully regenerate their limbs and tail anytime throughout life [1-4]. Contrary to urodeles, in anuran amphibians the capacity for appendage regeneration changes during ontogeny. While early tadpoles of the anuran species Xenopus laevis are capable of full limb and tail regeneration (except for a transient 'refractory' period during which the tail does not regenerate), the regenerative response decreases progressively with further development $[1,4,5]$. In consequence, early postmetamorphic Xenopus produce hypomorphic limb regenerates, and ultimately the regenerative ability is completely lost. In other anurans, a complete loss of the regenerative

We dedicate this paper to Professor Hermann Hartwig on the occasion of his 100th birthday, 1 January 2010.

\section{KARGER}

Fax +4161306 1234

E-Mail karger@karger.ch

www.karger.com (c) 2010 S. Karger AG, Basel

0304-324X/11/0571-0053\$38.00/0

Accessible online at:

www.karger.com/ger
Uwe Kierdorf

Department of Biology, University of Hildesheim

Marienburger Platz 22, DE-31141 Hildesheim (Germany)

Tel. +49 5121883 917, Fax +495121 883916

E-Mail uwe.kierdorf@uni-hildesheim.de 
ability occurs already at the late tadpole stage $[1,3,4]$. Regrowth of autotomized tails in lizards is another example of appendage regeneration in vertebrates. In this case an unsegmented cartilaginous tube is regenerated instead of a chain of vertebrae [1].

In contrast to the impressive regenerative abilities of 'lower' vertebrates, the capacity for appendage regeneration in mammals is generally poor. They are incapable of regenerating limbs, although limb buds of mice (Mus musculus) and rats (Rattus norvegicus) can regenerate [1, 6]. During further development, this regenerative capacity becomes, however, restricted to increasingly more distal regions and eventually to the tips of the digits $[1,7]$. Digit tip regeneration is also possible in humans, both in children and adults $[1,6,7]$. In humans and mice, digit regeneration occurs if amputation is through the distal portion of the terminal phalanx and if the wound is left open instead of being closed by suturing the skin over the amputation plane [7].

Deer antlers are a striking exception to the rule that mammals are incapable of full appendage regeneration $[8,9]$. Antlers are periodically replaced paired bony outgrowths that protrude from the foreheads of male deer and also of females in the reindeer (Rangifer tarandus). Antler renewal can be viewed as a kind of physiological regeneration, and the production of a new set of antlers each year proves that being an adult mammal does not preclude repeated regeneration of large appendages. Therefore, the study of antler renewal and its comparison with epimorphic regeneration in other vertebrates may provide crucial insights into why mammals are in general unable to regenerate appendages and show possible means how to overcome this inability. Understanding the mechanisms controlling antler regeneration may thus assist regenerative medicine to achieve its ultimate, but still distant, goal of inducing limb regeneration in humans [10].

The present paper summarizes the current knowledge on antler regeneration and compares this process with other examples of appendage regeneration in vertebrates. Particular attention will be given to aspects not or only cursorily dealt with in previous reviews [11-15] and to potential implications for regenerative medicine.

\section{Pedicle and Antler Formation}

Antlers develop as extensions from permanent bony frontal outgrowths referred to as pedicles [16]. Pedicle growth in young males is initiated and sustained by rising

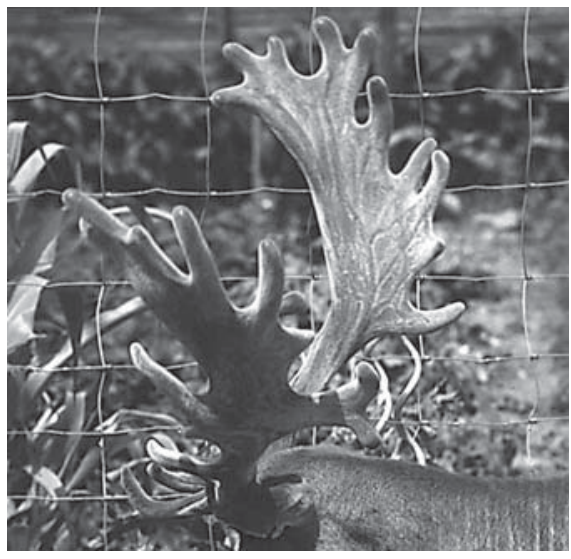

Fig. 1. Velvet-covered antlers of a fallow buck late during the antler growth period. Note the system of large blood vessels in the velvet.

blood testosterone levels. First antler growth from the pedicles starts spontaneously, when the latter are fully formed, and is associated with decreasing testosterone levels $[17,18]$. Because pedicle development is dependent on androgen action, castration early after birth prevents pedicle (and antler) formation [17]. The transition from pedicle to first antler growth is visible by a change of the integument covering the outgrowths from normal skin (pedicle) to a special type of pelage, known as velvet (fig. 1).

Antler growth is terminated by an increase in circulating testosterone that also causes full mineralization of antler bone and shedding of the velvet [17]. Along with the velvet, the antler's periosteum is removed, thereby exposing the bare bone of the antlers that are then referred to as 'hard antlers' (fig. 2). These are retained as long as blood testosterone concentrations stay above a certain threshold. When testosterone falls below this threshold after the rutting season, the antlers are dropped (antler casting). Starting after casting of the first antlers, a new set of antlers is periodically regenerated and lost from the pedicles. There is evidence that the effects of testosterone on the antlers are at least partly of an indirect nature, occurring after local conversion to estrogens $[12,17]$.

The first antlers of deer are small, unbranched spikes, whereas regenerated antlers, especially in larger species, successively increase in size and morphological complexity for several years. Antler growth is a very rapid process, the maximum rate of elongation recorded for wapiti (Cervus elaphus canadensis) antlers being $2.75 \mathrm{~cm}$ per day during mid-season [19]. Growing antlers are richly innervated by sensory fibers from branches of the trigeminal 


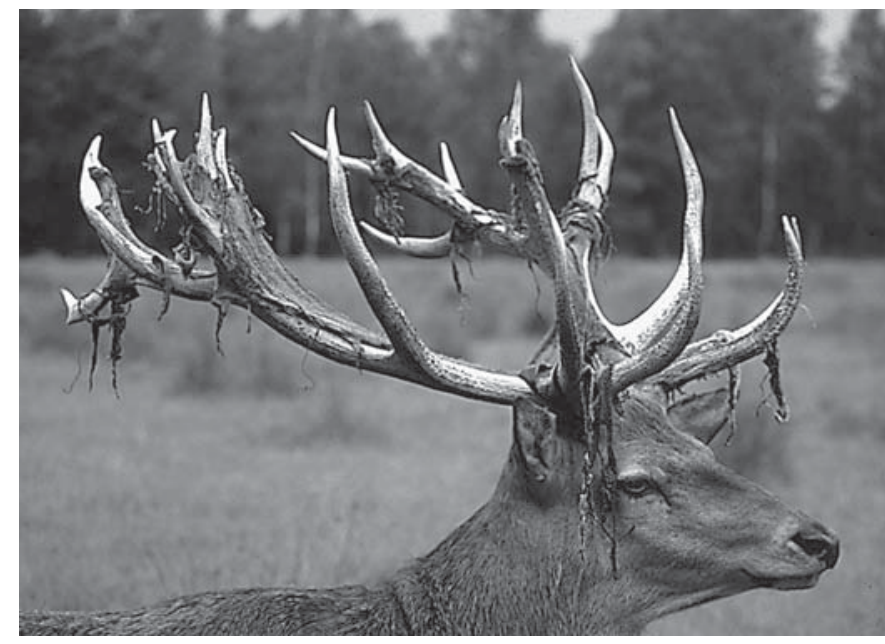

Fig. 2. Red deer stag at velvet shedding. The bare bone of the hard antlers is exposed.

nerve [8]. During antler renewal, new axons regenerate from the nerves in the pedicle. These regenerating nerve fibers grow out along with the major blood vessels, and a recent study suggested that secretion of nerve growth factor by the smooth muscle cells of the arteries and arterioles in the tip region of the growing antler may provide a guidance cue for the regenerating axons [20].

\section{Significance of the Antlerogenic Periosteum for Pedicle and First Antler Formation}

Deletion and transplantation experiments demonstrated that the capacity for pedicle and first antler growth resides in the frontal periosteum overlying the prospective pedicle site. Surgical removal of this 'antlerogenic periosteum' (AP) [8] in young deer prevents pedicle and antler formation, and autologous transplantation of AP (but not of periosteum from elsewhere on the frontals) to other sites causes ectopic pedicle and antler growth [21-23]. The ectopically formed antlers, which in extracranial sites were much smaller than normal antlers, were covered with velvet during growth and were regenerated in synchrony with the normal antlers (fig. 3) [21-24]. Rotation of the AP by $180^{\circ}$ caused a reversed anterior-posterior axis of the subsequently formed antlers, indicating that the AP carries morphogenetic information for axial orientation of the antler [25]. Since it has been shown that in mice the frontal bones are formed by cells of neural crest origin [26], it has been argued that

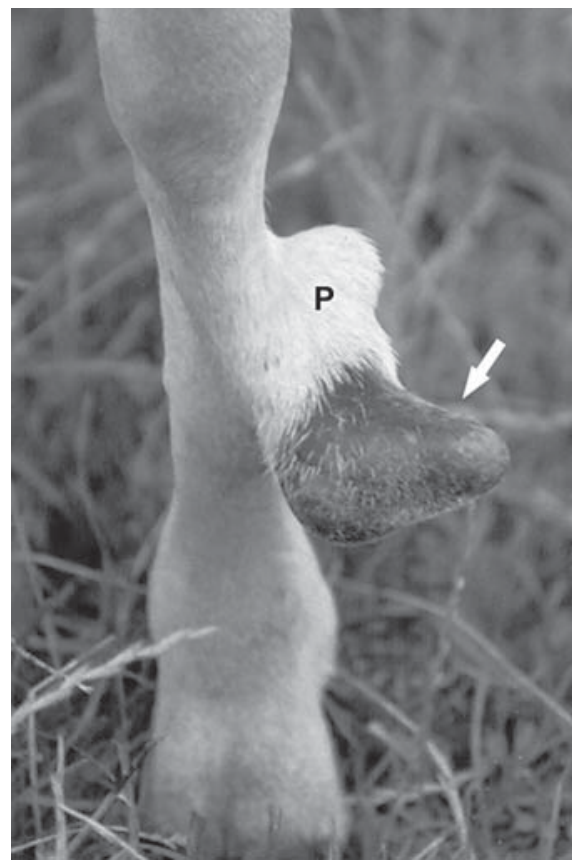

Fig. 3. Regenerating antler (arrow) on the foreleg of a fallow buck. Ectopic pedicle $(\mathrm{P})$ and antler growth were induced by autologous transplantation of AP.

the AP, pedicles, and antlers of deer are also neural crest derivatives [13].

The AP is much thicker than the periosteum from other cranial sites [27,28], and the cells of the cambium layer of the AP are rich in glycogen [15]. It has been suggested that the (cambium layer of the) AP contains stem cells and that pedicle and first antler growth depend on the activation of these stem cells $[14,15]$. It was shown that cells from the AP express the transcription factor gene OCT-4 (POU5F1) and that the expression of this gene is downregulated in cultured AP cells upon differentiation into adipocytes [29]. More recently it was reported that cells from the AP also express considerable levels of the cell surface antigen CD9 [15]. Cells isolated from the AP of red deer (Cervus elaphus) could be induced to differentiate along different (osteoblastic and adipogenic) mesenchymal lineages in vitro [29]. An in vivo study applying AP cell labeling with the lacZ gene at the time of pedicle initiation observed $\beta$-galactosidaseexpressing cells in mesenchyme, precartilage, cartilage, and bone (but not skin) of the growing appendage, thereby confirming the derivation of pedicles and first antlers from the AP [15].

Notwithstanding these achievements, there is a need for a more thorough characterization of the proliferation, 
maintenance, and differentiation potential of the AP cells. Growth of ectopic pedicles and antlers from transplanted AP clearly demonstrates that its cells are committed to a specific morphogenetic pathway and undergo autonomous differentiation. Furthermore, the regeneration of ectopic antlers indicates that the transplanted periosteal (stem) cells, or, more precisely, cells derived from them, persisted and proliferated in these locations.

That ectopic antlers are covered with velvet demonstrates that the competence for velvet differentiation is widespread in deer skin. Velvet transformation of skin is assumed to be induced by diffusible molecules produced by cells of the AP or their derivatives $[23,30]$. Support for this view comes from an experimental study in which insertion of an impermeable membrane between subcutaneously transplanted AP and overlying forehead skin prevented velvet transformation of the skin in male sika deer (Cervus nippon) fawns [31]. A recent investigation using the nude mouse model provided circumstantial evidence that the presumed inductive molecules are derived from the cambium (as opposed to the fibrous) layer of the AP [32].

\section{Hard Antlers, Antler Casting, and Wound Healing}

Most authors maintain that antlers die at or soon after velvet shedding due to an interruption of blood flow $[8$, $33,34]$. During growth, the antler is supplied with blood mainly by arteries located in the vascular layer of the velvet (fig. 1). Initially, the venous drainage of the antler predominantly takes place internally through veins of the pedicle. However, when the vascular channels in the antler bone become progressively narrowed due to ongoing bone deposition during later stages of antler growth, blood flow through the antler's interior is dramatically diminished and the venous drainage of the antler occurs chiefly through blood vessels in the velvet [35]. When these peripheral vessels are removed at velvet shedding, sufficient blood circulation in the antler cannot be maintained and the antlers die off $[34,35]$.

Some investigators, however, argue that sufficient blood circulation to keep them viable exists in antlers for months after velvet shedding through internal vessels connecting the pedicle with the antler [36]. According to this view, death of the hard antlers is eventually caused by a reduction of internal blood supply late in the hard antler phase.

The hypothesis that hard antlers are living structures does not account for the fact that premature casting of hard antlers, but not of antlers in velvet, can be induced by castration or antiandrogen treatment $[8,17,37]$. These findings suggest that hard antlers are actually 'ready' to be cast, i.e. that they are dead structures, but that the osteoclastic activity causing antler casting is prevented by higher blood levels of testosterone. This view is supported by the observation that antler casting is prevented in individuals whose testosterone levels are artificially elevated. The same effect can be achieved if estrogen instead of testosterone is administered [38].

Antler casting is preceded by osteoclastic activity that is most intense in a narrow zone of the distal pedicle corresponding to the future separation plane $[33,39]$. Bone resorption occurs both within the pedicle and at its surface $[33,39,40]$. Typically, the antler plus a small portion of the most distal pedicle bone breaks away from the pedicle before all connections between the two parts have been eroded. The base of the cast antler and the breakage plane of the pedicle therefore exhibit an uneven texture with bony spicules $[8,40]$.

Antler casting is followed by some bone restoration in the pedicle that partly compensates the bone loss occurring at antler casting [33, 34, 41, 42]. As this compensation is, however, only a partial one, the pedicles decrease in height with age. On the other hand, they increase in thickness throughout life due to periosteal bone apposition.

By antler casting a bone wound forms at the distal end of the pedicle (fig. 4). Healing of the skin over of this wound reestablishes the integumental continuity that was disrupted at velvet shedding. Antler regeneration does, however, also occur when the previous antler is not cast and no casting wound is formed. In this case, the new antler growth leads to the formation of an abnormality known as 'double-head antler', i.e. of two successively grown antlers emanating from one pedicle (fig. 5) [40, 43]. Double-head antlers demonstrate that antler regeneration is not caused by the loss of the old antlers. Rather, antler casting and renewal are presumably reactions to the same stimulus, namely a drop in circulating androgen levels $[40,43]$. It has been argued that antler regeneration is triggered by the death of the antlers (occurring after velvet shedding), but that the onset of the regenerative process is suppressed by higher levels of circulating androgens [40]. In some deer species, there is a time lag between antler casting and onset of new antler growth, indicating the latter's dependence on a (photoperiodically controlled) growth stimulus, the nature of which is controversial $[8,17]$. There is, however, evidence from in vivo and in vitro studies that insulin-like growth factor-I 


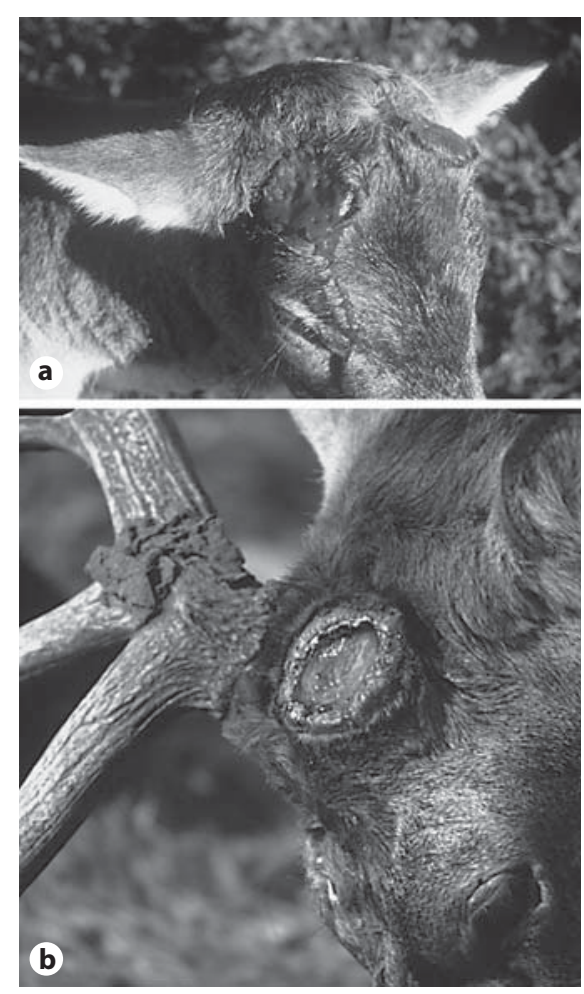

Fig. 4. Casting wounds in a fallow buck (a) and a red deer stag (b). Bleeding from the pedicle wounds early after casting (a) and onset of epithelialization of the wound area from the peripheral skin rim at a slightly later stage (b). b As is demonstrated, antler casting can be asynchronous between sides.

is the main hormone with a stimulating effect on antler growth $[11,12]$.

Healing of the casting wound on the pedicle stump is not as well understood as are later stages of antler regeneration, and there is currently no consensus about even very basic aspects of this process. In principle, the mode of wound healing following loss or removal of an appendage in a vertebrate differs fundamentally between regenerating and nonregenerating appendages [44-46]. In the latter case, a fibrotic scar is formed and it has been suggested that this scar precludes the formation of a regeneration blastema and thus appendage regeneration by blocking the necessary signaling between wound epidermis and the underlying stump tissues [45-47]. According to this view, avoiding or minimizing scar formation during wound healing is a necessary prerequisite for successful appendage regeneration.

Goss [8, 10,47], one of the most eminent antler biologists of the 20th century, has repeatedly stressed that antler regeneration is a very special process because, in con-

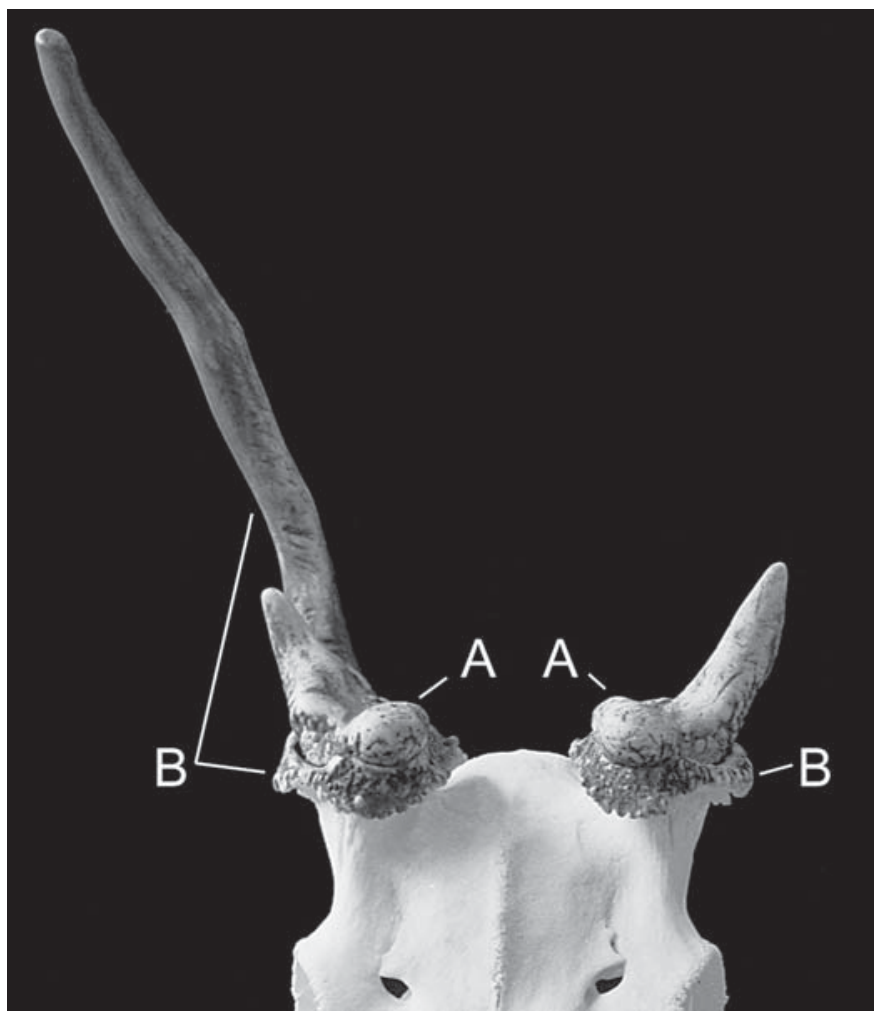

Fig. 5. Bilateral double-head antlers in a fallow buck. $A=$ Undetached first antlers; $\mathrm{B}=$ regenerated antlers.

trast to other wound healing processes in adult mammals, the formation of a scar is avoided. Recently, however, it has been stated that healing of the casting wound regularly involves scarring of varying intensity [15].

Following antler casting, the breakage plane of the pedicle is covered with blood originating from ruptured vessels of the pedicle (fig. 4a). Bleeding is, however, not profuse and soon the exposed pedicle bone is covered with a blood clot that, upon drying of the wound, forms a scab. In our experience, what appears to be a small scar can sometimes occur in the region where this scab persists longest during wound healing. In species like red deer and fallow deer (Dama dama), this is the bifurcation area between the main beam and the brow tine. Thus, we conclude that casting wound healing can be either scarfree, which is regarded as the more typical outcome of the healing process, or involve minor scarring. Interestingly only minimal scarring is also observed in regenerating human fingertips [7]. 
During typical skin wound healing in adult mammals, the initially formed granulation tissue is transformed into the scar tissue $[46,48]$. A capillary-rich fibrocellular tissue resembling granulation tissue has also been reported to occur between the pedicle bone and the scab during casting wound healing $[39,41,42]$. The fate of this tissue has not been studied so far, but it is assumed that if a scar is formed during antler regeneration it is derived from it.

The special nature of the integumental response in antler regeneration is further evidenced by the fact that numerous new hair follicles are formed in the velvet that covers the regenerating antler [8]. The mechanisms involved in follicular neogenesis during antler regeneration are unknown. Interestingly, neogenesis of hair follicles has recently also been described in healing skin wounds of genetically normal adult mice and has been shown to be dependent on Wnt/ $\beta$-catenin signaling [49].

Contrary to adults, mammalian embryos and early fetuses heal cutaneous wounds without scarring and regenerate a normal skin structure [50]. A principal difference between skin wound healing in embryonic and fetal mammals compared with adult individuals is the degree of inflammation involved. Fetal skin wound healing is associated with very little inflammation, whereas healing of cutaneous wounds in adult mammals typically involves a strong inflammatory response $[46,50,51]$. We hypothesize that the absence or low degree of scarring during healing of casting wounds may be due to a local suppression of inflammation.

The available, purely histological evidence indeed suggests that the inflammatory response in the casting wound is not very intense [33]. The study of expression profiles of signaling molecules (especially pro- and antiinflammatory cytokines) during casting wound healing and the analysis of experimental alterations of these patterns, therefore, appear as promising fields for future studies. It would be particularly interesting to investigate whether it is possible to induce major scarring during healing of the casting wound and thereby impair or even inhibit antler regeneration.

Already before antler casting, the skin in the most distal pedicle region acquires the typical features of antler velvet. Starting from this skin rim, epidermal cells move over the wound area, leading to its epithelialization (fig. 4b) [41]. The migrating epidermis dissects through the wound area by moving underneath the scab [34, 41], as has also been observed in other cases of mammalian skin wound healing [48]. Electron microscopy and immunohistochemistry demonstrated early formation of a basal lamina during antler regeneration. This basal lam- ina is established in peripheral areas of the healing casting wound at a time when epithelialization of the more central wound areas has not yet been completed $[13,15]$. Histological studies further showed that the epidermal ingrowth is accompanied by that of the dermis so that, within a matter of days, the casting wound area is overlain by a layer of skin rather than just a wound epidermis $[39,42]$.

Healing of full-thickness skin over the pedicle stump, rather than covering of the wound surface with a wound epidermis, is a potentially important difference from epimorphic regeneration of other vertebrate appendages. In regenerating amphibian limbs as well as in regenerating zebrafish (Danio rerio) fins the amputation wound is rapidly (within a few hours) covered by the wound epidermis, which then thickens to form the so-called apical epidermal cap (AEC) $[2,52]$. Wound epidermis and AEC are of critical importance for the formation of the regeneration blastema and the proliferation of its cells. Removal of the AEC or covering of the amputation stump with full-thickness skin inhibits limb regeneration in amphibians $[1-3,44]$. The necessary short-range signaling between epidermal cells and those of the underlying stump/ blastema requires prolonged close approximation between them, and, in contrast to antler regeneration, a new basal lamina is formed late during amphibian limb regeneration $[2,53]$. The nature of the signaling between wound epidermis and underlying blastema is not well understood; however, expression patterns of fibroblast growth factor $(F G F)$ genes in regenerating amphibian limbs suggest that FGFs are important for the control of blastema survival and growth $[1,3]$. FGFs are probably also involved in the control of antler growth. Thus, FGF-2 has been demonstrated to increase the proliferation rate of cultured cells from the mesenchymal growth zone of the regenerating antler [11] and a microarray study indicated that FGF signaling is activated in the antler mesenchyme [54].

Covering of the wound area with full-thickness skin inhibits postamputational digit tip regeneration in mammals $[1,6]$. A corresponding result has also been reported for antler regeneration [45]. In this experiment the distal half of the pedicle was surgically removed and the pedicle skin folded back over the pedicle stump. This caused a complete inhibition of antler growth (1 case) or led to the production of stunted antlers (4 cases). The outcome of the experiment was interpreted as indicating that skin wound healing is a prerequisite for antler regeneration. However, the results would also be compatible with the assumption that pedicle amputation removed (most of) 
the periosteal cells responsible for antler regeneration (see below).

It has recently been demonstrated that antler regeneration is not blocked when the skin is prevented from healing over the wound [55]. In this experiment the pedicle was cut just below the antler-pedicle junction. The skin was mechanically separated from the pedicle bone and an impermeable membrane inserted between skin and bone. This intervention did not inhibit a regenerative response from the pedicles. The bony outgrowths that developed from the amputation stumps lacked a skin cover, their surface instead being covered with a scab that was underlain by a tissue resembling granulation tissue. The skinless outgrowths from the pedicle stumps were shorter than the velvet-covered antlers growing from the sham-operated contralateral pedicles; however, one of the skinless outgrowths even exhibited a rudimentary branching. This led the authors to conclude that the structures were actually regenerated antlers and not just extensions from the pedicle bone. The stunted growth of the 'skinless antlers' was attributed mainly to insufficient blood supply [55]. It is currently unknown whether such antler structures can be cast and regenerated. The histological and experimental findings indicate that a direct contact between wound epidermis and underlying pedicle tissue is not a necessary prerequisite for antler regeneration. Thus, contrary to other examples of appendage regeneration in vertebrates, antler regeneration is apparently not strictly dependent on a wound-healing process and on signaling between wound epidermis and the underlying tissue.

\section{Histo- and Morphogenesis of the Regenerating Antler}

Key questions in regenerative biology are those of the origin, lineage specification and fate of the cells from which a regenerated structure develops. The origin of the cells that build up the regenerating antler has long been a matter of controversy. On the basis of histological studies it was originally believed that these cells are derived from the pedicle dermis $[19,34]$. However, it was later shown that antler regeneration can occur when the skin is not involved, leading to the formation of (stunted) 'skinless antlers' [55]. In contrast, our group suggested that the regenerating antler is formed by cells originating from the pedicle periosteum (PP) $[40,41,43]$. This view was initially based on the morphological analysis of double-head antlers, demonstrating that when the old antler is not

Deer Antlers: A Model of Mammalian

Appendage Regeneration cast, the regenerated antler develops as a periosteal exostosis from the distal pedicle [56]. Histological studies of normal antler regeneration later showed that early after antler casting the periosteum of the distal pedicle is markedly thickened, which is suggestive of a high proliferative activity in the distal PP $[41,42]$. Furthermore, it was demonstrated that the mesenchymal growth zones of the main beam and the brow tine of red deer form very early during the regeneration process by a local thickening of the PP [42]. That cells of the PP are responsible for antler regeneration has also been supported experimentally [57]. Thus, surgical removal of the PP in red deer stags either inhibited antler regeneration throughout the following antler growth season or delayed the process until after the PP had regenerated. Since in this experiment the pedicles had been cut about $1 \mathrm{~cm}$ below the antler/ pedicle junction and the cut surface sealed with bone wax, participation of cells derived from the pedicle bone proper (as opposed to the $\mathrm{PP}$ ) in the regeneration process could be largely excluded [57].

Following limb amputation in urodeles, the skeletal elements in the distal stump undergo marked histolysis and the tissues lose their mature histological characteristics. It has been demonstrated that expression of matrix metalloproteinases (MMPs), especially MMP9, is upregulated early after amputation and it has been postulated that MMPs play a major role in both matrix degradation and the formation of the wound epidermis $[44,53]$.

Reversal of cells to a less-differentiated state is known as dedifferentiation. In urodele limbs, proliferation of dedifferentiated cells leads to the accumulation of a mass of undifferentiated cells, the regeneration blastema, at the end of the amputation stump [1-3, 44]. It is, however, discussed whether also (as yet unidentified) quiescent stem cells in the dermis are activated and contribute cells to the blastema [3].

The extent of dedifferentiation by the cells in the amphibian limb stump has long been debated, as was the question of whether the blastema cells are able to redifferentiate into cell types that differ from those of their tissue of origin (transdifferentiation) [1-3]. Traditionally, the blastema of the regenerating amphibian limb was considered a more or less homogeneous group of multior pluripotent cells capable of differentiating along several lineages [1]. However, there is increasing evidence that this is not the case and that the dedifferentiation process is less complete than was previously thought [3]. A recent study using an integrated green fluorescence protein (GFP) transgene to track cell fates during limb regeneration in the axolotl (Ambystoma mexicanum) 
demonstrated that the blastema cells deriving from marked limb cells differentiated along restricted lineages according to their tissue of origin [58]. Thus, e.g. blastema cells derived from GFP+ dermis cells formed connective tissue, cartilage and tendons, but not myogenic cells or Schwann cells. The findings of this study indicate that from the outset the blastema of the regenerating axolotl limb is a heterogeneous collection of cell-lineage-restricted progenitor cells rather than a mass of pluripotent cells [58].

There is evidence suggesting that muscle formation in regenerating urodele limbs may also involve the activation of resident stem cells. Thus, Pax7+ satellite cells, which are comparable to mammalian skeletal muscle stem cells, were observed in limb muscle and, following amputation, also in the regeneration blastema of the redspotted newt (Notophthalmus viridescens). This led to the hypothesis that quiescent Pax7+ stem cells are activated in the limb stump and contribute progenitor cells to the regeneration blastema [59].

Interestingly, the mechanisms leading to tail regeneration appear to differ between urodeles and larval anurans [44]. In urodeles, the tail blastema forms from dedifferentiated stump cells, and in the larval axolotl the major cell contribution to this blastema is from dedifferentiating muscle fibers $[44,60]$. In contrast, using GFP-labeling it was shown that in Xenopus tadpoles the myofibers of the regenerate were derived from Pax7+ satellite cells, and that the spinal cord and the notochord regenerated not from a blastema, but from the same tissue types in the stump with no exchange of cells with other compartments and no dedifferentiation or transdifferentiation [61].

The origin of the regeneration blastema of the zebrafish fin is currently not definitely known; the available data, however, suggest that it is formed from dedifferentiated cells. Pigment cells are an exception, since they have been proposed to be stem cell derived [52, 62]. The cell source of the blastema that has recently been reported to form during mammalian digit tip regeneration is unknown [7].

In the case of antler regeneration, histological studies revealed no histolysis or other indications of appreciable dedifferentiation processes in the distal pedicle after antler casting $[33,41,42]$. Also, no MMP9 signal was detectable in healing casting wounds by in situ hybridization [15]. This suggests that the mesenchymal growth zones of the regenerating antlers are not formed from dedifferentiated pedicle stump cells. Instead, as has been discussed, there is evidence that the antler growth zones are derived by proliferation from a local cell source, namely the PP $[41,42]$.

At present, antler regeneration is considered a stem cell-based process [13-15], a view that is based on different lines of evidence. Thus, studies in red deer and fallow deer have demonstrated that the PP contains cells expressing different stem cell markers (CD9, OCT-4, Nanog, STRO-1) $[15,63]$. It has also been reported that PP cell populations show elevated activity of the enzyme telomerase [15]. Furthermore, it was shown that in vitro cells from the PP can be induced to differentiate along different mesenchymal (osteogenic, chondrogenic, adipogenic) lineages, thereby indicating their multipotency $[15,64]$. Cells from the AP, which are assumed to give rise to those of the PP $[13,16]$, were recently reported to differentiate into muscle precursor cells and cells resembling neurons upon cultivation in appropriate media [15]. That AP cells are probably able to differentiate into nerve cells is not surprising given the presumed derivation of the AP from the neural crest. The observation that PP cells express neural crest cell markers [64] supports the view of a neural crest derivation of $\mathrm{AP}$ and $\mathrm{PP}$.

That cells from the PP can be induced to differentiate along several lineages in vitro does, however, not mean that all of these differentiation pathways are also followed in vivo [13]. Further studies are needed to investigate the relationship between the developmental potential and the actual in vivo differentiation of the PP-derived cells.

In summarizing our current knowledge, it has been suggested that antler regeneration can be characterized as a stem cell-based dedifferentiation-independent epimorphic process that depends on the periodic activation of stem cells located in the PP $[15,16]$. To allow periodic antler regeneration throughout the life of a deer, the stem cell pool in the PP must be maintained by lifelong selfrenewal. It is suggested that the PP stem cells give rise to progenitor cells (no longer capable of self-renewal) whose proliferation then leads to the formation of the antler's mesenchymal growth zones. The mesenchymal progenitor cells from these zones have an extended life span in vitro. They could be grown for over 80 passages and for up to 10 months before stopping to divide [65].

It is a matter of controversy whether the richly vascularized mesenchymal growth zones present at the tips of the main beam and tines of growing antlers $[66,67]$ can be equated to blastemas of other regeneration systems [13]. Using the proliferation marker PCNA it was demonstrated in red deer that the mesenchymal growth zone can be subdivided into an outer (distal) portion with a lower and an inner (proximal) portion with a higher pro- 
portion of dividing cells [67]. Interestingly, a similar observation has been made in the regeneration blastema of the caudal fin of zebrafish [62]. During the outgrowth phase, cells in the proximal blastema proliferate at a high rate, whereas those of the distal blastema show no or very slow proliferation. The functional significance of this subdivision of the growth zone/blastema remains to be established in the case of both antler and fin regeneration.

Proximally, each antler growth zone is followed by a zone of differentiation, populated mainly by chondroprogenitors [66, 67]. The chondroblasts and chondrocytes sequentially differentiating from these progenitors become arranged in preferentially vertically oriented columns that are separated by vascular spaces. Further proximally, the matrix of the cartilaginous trabeculae is mineralized and the chondrocytes undergo hypertrophy and apoptosis. The mineralized cartilage is then eroded by chondroclasts and bone is laid down on the scaffold provided by the remnants of the cartilaginous trabeculae. Eventually, the cartilage is completely replaced by bone $[12,33,66$, 68]. In addition to this endochondral ossification, also direct (subperiosteal) bone formation has been reported to occur in antlers of different deer species, the appositional growth leading to the formation of a peripheral bone sleeve $[12,13,66,68]$. Mature antlers consist of a central portion of cancellous bone and an outer layer (cortex) of compact bone, with a narrow transition zone between the two portions [66]. Formation of a dense antler cortex is apparently androgen-dependent as is indicated by its lack in castrates [69].

Our understanding of the factors/signaling pathways involved in the local control of antler growth is still in its infancy $[11,14]$. As already mentioned, FGF signaling appears to be involved in the control of proliferation of the progenitor cells from the mesenchymal growth zone [11, 54]. A study investigating the distribution of the de-phosphorylated form of $\beta$-catenin by immunocytochemistry found the most intense staining in this zone [70], suggesting a role for Wnt $/ \beta$-catenin signaling in antler growth. More specifically, it has been concluded that $\beta$-catenin is an important factor controlling survival and lineage specification of the mesenchymal progenitor cells [70]. Wnt/ $\beta$-catenin signaling is known to be required for blastema formation in amphibian limb and zebrafish fin regeneration and to play an important role also during the outgrowth phase of fin regeneration [44].

The retinol derivative retinoic acid (RA) is involved in the control of pattern formation in different developing systems of vertebrates. RA is also present in the blastema of the regenerating amphibian limb, and excess
RA applied to the early regeneration blastema changes the positional identities of the blastema cells in a dosedependent way, causing the formation of abnormal regenerates $[1,4]$.

There is evidence that RA is also involved in the regulation of cellular differentiation during antler growth, particularly in chondro- and osteogenesis. Thus, significant amounts of retinol were found in antler tissues at all stages of differentiation and the RA-synthesizing enzyme RALDH2 was immunolocalized in skin, perichondrium, perivascular cells in cartilage, and in osteoblasts at sites of bone formation in regenerating red deer antlers [71]. All-trans-RA and 4-oxo-RA were found in skin, perichondrium, cartilage, bone, and periosteum, while 9-cisRA was present in perichondrium, mineralized cartilage and bone. RAR $\alpha$ (receptor for all-trans-RA) and RXR $\beta$ (receptor for 9-cis-RA) were both expressed in skin and perichondrium, whereas RXR $\beta$ was specifically expressed in chondrocytes and RAR $\alpha$ in perivascular cells [71]. A role for RA in antler osteogenesis is also indicated by the fact that in vitro RA increased the expression of alkaline phosphatase (a marker of osteoblast differentiation) by mesenchymal cells from the antler growth region. There exists also some in vivo evidence from studies in fallow deer suggesting that antler growth and morphogenesis can be influenced by exogenous RA $[72,73]$.

The morphology of antlers is species-specific and, to a certain extent, also age-specific and it would be interesting to know how this specificity is achieved. However, except for the potential role of RA as a local factor, very little is known about the control of antler shape. Although antler growth per se does not require a functional nerve supply [74], it has been hypothesized that the nervous system is involved in the control of antler morphogenesis [75]. This view is based on observations on the effects of trauma to growing antlers. Following injury, specific growth deviations, such as the formation of additional tines, can occur. It has been reported that occasionally these extra tines re-appear during subsequent antler cycles before the antler morphology eventually reverts to normal. The phenomenon has been named 'trophic memory' and the effect is said to occur only if the animals (their antlers) are not anesthetized when the trauma happens [75]. Should the phenomenon of trophic memory, and thus the involvement of the nervous system in the control of morphogenesis, be substantiated in future studies, antlers could be a model for pattern formation by a combination of local and remote (long-range) control mechanisms. 
Fig. 6. Hard first antler of a fallow buck showing an osteochondroma attached to the antler base.

Fig. 7. Skin-covered antlers of a castrated fallow buck showing bony protuberances.

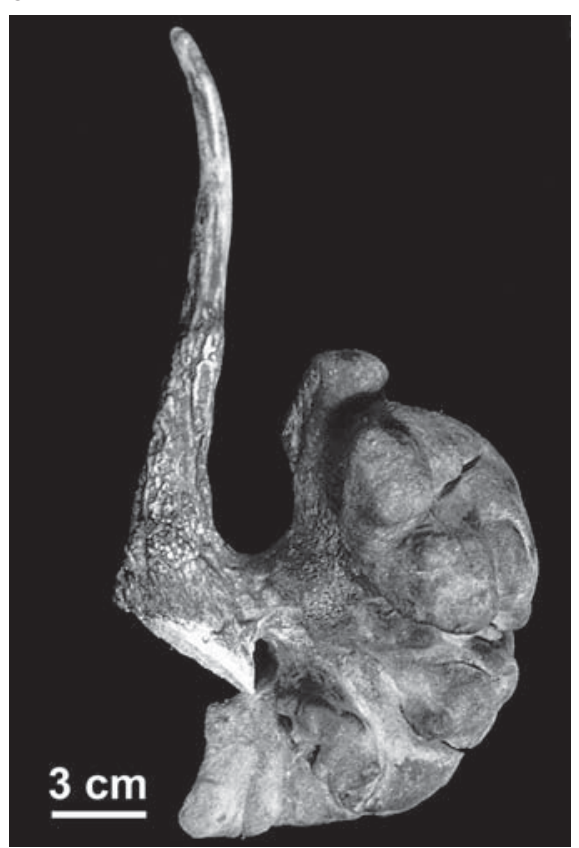

7

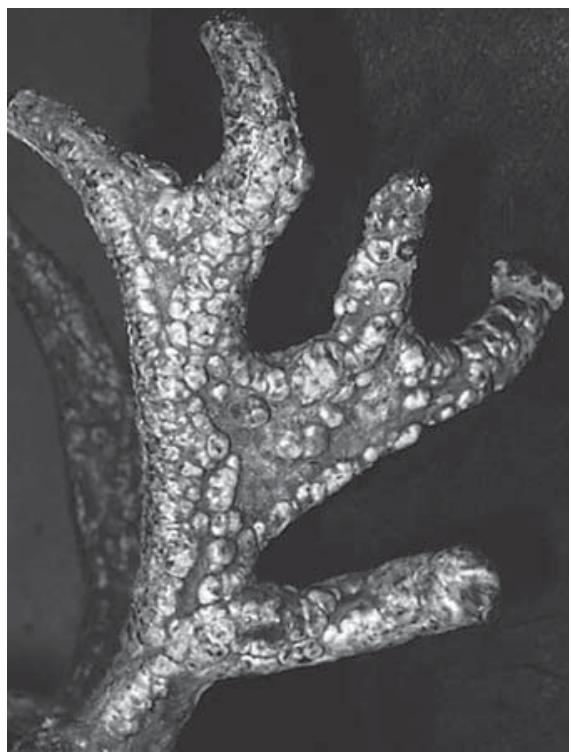

\section{Antler Growth and Tumor Formation}

The enormous growth rate of antlers has repeatedly led authors to ask whether these structures are susceptible to tumor formation $[8,47]$. One author even suggested that normal antlers are comparable to malignant tumors and that annual antler casting evolved as a means to protect the bearer of the antlers from damage [76]. This view must be rejected, since antler formation is a well-coordinated developmental process showing none of the growth abnormalities associated with malignant tumors. In fact, growing antlers appear to be very resistant to tumor formation $[8,47]$. Thus, to our knowledge so far only one example of an antler bone tumor in an intact male deer has been described. This tumor was diagnosed as an osteochondroma (fig. 6) [77].

It has been argued that the (virtual) lack of tumors in antlers of intact males can be attributed to the fact that their antlers remain viable for only some months, which is shorter than the time required by most tumors to become overt [47]. However, an apparent lack of spontaneous tumorigenesis is also observed in regenerating urodele limbs that, moreover, show a low frequency of tumor development upon exposure to carcinogens [78]. It has, therefore, been argued that the capacity for epimorphic regeneration is associated with a resistance to tumor formation [79]. The similarity in tumor resistance between regenerating antlers and urodele limbs is highly interest- ing, not least because of the different origin of the regenerative tissue in the two systems. Colitti et al. [67] observed a high rate of apoptosis in the antler mesenchymal growth zone. They suggested that programmed cell death in growing antlers may be involved in pattern formation (delineating the position of branches) but also function to prevent tumor development by eliminating transformed cells.

When a male deer carrying hard antlers is castrated, its antlers are prematurely cast and a new set of antlers is produced. These remain permanently viable, because the sex steroids necessary for bone maturation and velvet shedding are missing. Likewise, when castration takes place during the antler growth phase, the velvet-covered antlers will be permanently retained on the animal's forehead [8]. Interestingly, the antlers of castrates show a tumor-like overgrowth of bone and skin, the intensity of which differs between species.

Thus far, the histology of such 'castrate antlers' has been studied in detail only in the fallow deer. These studies suggested that the abnormal skin outgrowths, which were termed antleromas [8], represent a hypertrophy of the dermis $[69,80]$. The bony protuberances present in antlers of castrates are formed by appositional growth from the periosteum (fig. 7) [69]. Both the skin and bone overgrowths of fallow deer can be classified as benign tumors, and malignant transformation of these structures has not been observed $[69,80]$. Also in the roe deer, which 
shows the most exuberant antler growth following castration, causing the formation of so-called peruke antlers, malignant characteristics such as invasiveness and metastasis are missing [8]. Moreover, the antlers of castrated deer maintain a seasonal growth pattern, and mineralization of antler bone and velvet shedding can be induced by application of larger doses of testosterone [8]. The available evidence thus suggests that both the shortlived antlers of intact males and the long-lived antlers of castrates are resistant to the formation of malignant tumors.

The ultimate goal of regenerative medicine, namely inducing the regeneration of normally nonregenerating structures, is associated with the risk that the proliferative response gets out of control. Studying antlers may provide essential insights into the developmental control of rapid regenerative growth and thereby help to avoid detrimental side effects of induced regeneration.

\section{Conclusion and Outlook}

The periodic renewal of antlers is an impressive example of appendage regeneration in an adult mammal. Antler regeneration involves the formation of a mass of mesenchymal cells as is also the case in other systems of epimorphic regeneration in vertebrates, and there is evidence for considerable overlap in the developmental pathways activated in the regeneration processes across various taxa. However, there are also important differences between the different systems of epimorphic regeneration, such as the origin of the cells that form the regen- erating structure. Thus, there is accumulating evidence that the regenerating antler is built up by progenitor cells originating from a specific source, namely stem cells in the PP. In contrast to the situation in amphibian limb regeneration, the process of antler regeneration does not involve appreciable cell dedifferentiation.

For different reasons, a deeper understanding of antler regeneration can be important for regenerative medicine. Thus, studying the mechanisms involved in the healing of the casting wound may provide clues for reducing scarring during wound healing in humans. In addition, a more profound understanding of the molecular mechanisms controlling antler renewal could lead to new therapeutic approaches in stimulating limb regeneration. It has been suggested that the challenge of inducing limb regeneration in humans is to find a way to induce fibroblast dedifferentiation in the stump [6]. This assumes that if limb regeneration could be induced in mammals, it would use mechanisms similar to those operating in amphibian limbs. This assumption may be true and is clearly a reasonable starting point for further research. However, antlers demonstrate that appendage regeneration in a mammal can also occur in a different way, suggesting that it may be worthwhile to also consider other approaches to stimulate a regenerative response. Finally, the antlers are of medical interest as a model for studying how a structure undergoing rapid regenerative growth can escape cancer development. In our view, the above prospects fully justify an intensification of studies on antler regeneration despite the obvious challenges posed by using deer as experimental animals.

\section{References}

1 Stocum DL: Regenerative Biology and Medicine. Amsterdam, Elsevier Academic Press, 2006.

2 Carlson BM: Principles of Regenerative Biology. Amsterdam, Elsevier Academic Press, 2007.

3 Gardiner DM, Bryant SV: Tetrapod limb regeneration; in Hall BK (ed): Fins into Limbs: Evolution, Development, and Transformation. Chicago, University of Chicago Press, 2007, pp 163-182.

4 Yakushiji N, Yokoyama H, Tamura K: Repatterning in amphibian limb regeneration: a model for study of genetic and epigenetic control of organ regeneration. Semin Cell Dev Biol 2009;20:565-574.

\footnotetext{
5 Tseng AS, Levin M: Tail regeneration in Xenopus laevis as a model for understanding tissue repair. J Dent Res 2008;87:806-816.

6 Gardiner DM: Ontogenetic decline of regenerative ability and the stimulation of human regeneration. Rejuvenation Res 2005;8:141153.

-7 Han M, Yang X, Lee J, Allan CH, Muneoka $\mathrm{K}$ : Development and regeneration of the neonatal digit tip in mice. Dev Biol 2008;315: 125-135.

8 Goss RJ: Deer Antlers: Regeneration, Function, and Evolution. New York, Academic Press, 1983.

9 Goss RJ: Epimorphic regeneration in mammals; in Hunt TK, Heppenstall RB, Pines E, Rovee D (eds): Soft and Hard Tissue Repair.

10 Goss RJ: Prospects for regeneration in man. Clin Orthop Relat Res 1980;151:270-282.

-11 Price J, Faucheux C, Allen S: Deer antlers as a model of mammalian regeneration. Curr Top Dev Biol 2005;67:1-48.

12 Price JS, Allen S, Faucheux C, Althnaian T, Mount JG: Deer antlers: a zoological curiosity or the key to understanding organ regeneration in mammals? J Anat 2005;207:603618.

13 Kierdorf U, Kierdorf H, Szuwart T: Deer antler regeneration: cells, concepts, and controversies. J Morphol 2007;268:726-738.

14 Kierdorf U, Li C, Price J: Improbable appendages: deer antler renewal as a unique case of mammalian regeneration. Semin Cell Dev Biol 2009;20:535-542.
} New York, Praeger, 1984, pp 554-573. 


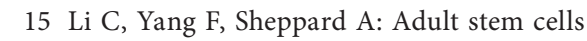
and mammalian epimorphic regeneration insights from studying annual renewal of deer antlers. Curr Stem Cell Res Ther 2009; $4: 237-251$.

-16 Kierdorf U, Kierdorf H: Pedicle and first antler formation in deer: anatomical, histological, and developmental aspects. Z Jagdwiss 2002;48:22-34.

17 Bubenik GA: The endocrine control of the antler cycle; in Brown RD (ed): Antler Development in Cervidae. Kingsville, Caesar Kleberg Wildlife Research Institute, 1983, pp 73-107.

18 Suttie JM, Lincoln GA, Kay RNB: Endocrine control of antler growth in red deer stags. J Reprod Fertil 1984;71:7-15.

19 Goss RJ: Problems of antlerogenesis. Clin Orthop Relat Res 1970;69:227-238.

-20 Li C, Stanton JL, Robertson TM, Suttie JM, Sheard PW, Harris AJ, Clark DE: Nerve growth factor mRNA expression in the regenerating antler tip of red deer (Cervus elaphus). PLoS One 2007;2:e148.

-21 Hartwig H, Schrudde J: Experimentelle Untersuchungen zur Bildung der primären Stirnauswüchse beim Reh (Capreolus capreolus L.). Z Jagdwiss 1974;20:1-13.

22 Goss RJ, Powel RS: Induction of deer antlers by transplanted periosteum. I. Graft size and shape. J Exp Zool 1985;235:359-373.

23 Kierdorf H, Kierdorf U: The role of the antlerogenic periosteum for pedicle and antler formation in deer; in Sim JS, Sunwoo $\mathrm{HH}$, Hudson RJ, Jeon BT (eds): Antler Science and Product Technology. Edmonton, ASPTRC, 2001, pp 33-51.

24 Goss RJ: Induction of deer antlers by transplanted periosteum. II. Regional competence for velvet transformation in ectopic skin. J Exp Zool 1987;244:101-111.

25 Goss RJ: Induction of deer antlers by transplanted periosteum. III. Orientation. J Exp Zool 1991;259:246-251.

26 Jiang XB, Iseki S, Maxson RE, Sucov HM, Morriss-Kay GM: Tissue origins and interactions in the mammalian skull vault. Dev Biol 2002;241:106-116

-27 Kierdorf H, Kierdorf U, Szuwart T, Gath U, Clemen G: Light microscopic observations on the ossification process in the early developing pedicle of fallow deer (Dama dama). Ann Anat 1994; 176:243-249.

28 Li C, Suttie JM: Light microscopic studies of pedicle and early first antler development in red deer (Cervus elaphus). Anat Rec 1994; 239:198-215.

-29 Berg DK, Li C, Asher G, Wells DN, Oback B: Red deer cloned from antler stem cells and their differentiated progeny. Biol Reprod 2007;77:384-394.

30 Goss RJ: Of antlers and embryos; in Bubenik GA, Bubenik AB (eds): Horns, Pronghorns, and Antlers. New York, Springer, 1990, pp 298-312.
31 Li C, Yuang F, Xing X, Gao X, Deng X, Mackintosh C, Suttie JM: Role of heterotypic tissue interactions in deer pedicle and first antler formation - revealed via a membrane insertion approach. J Exp Zool (Mol Dev Evol) 2008;310B:267-277.

32 Li C, Gao X, Yang F, Martin SK, Haines SR, Deng X, Schofield J, Stanton JL: Development of a nude mouse model for the study of antlerogenesis - mechanism of tissue interactions and ossification pathway. J Exp Zool (Mol Dev Evol) 2009;312B:118-135.

33 Gruber GB: Morphobiologische Untersuchungen am Cerviden-Geweih. Werden, Wechsel und Wesen des Rehgehörns. Nachr Ges Wiss Göttingen Math Phys Kl NF Fachgr VI 1937;3:9-63.

34 Wislocki GB: Studies on the growth of deer antlers. I. On the structure and histogenesis of the antlers of the Virginia deer (Odocoileus virginianus borealis). Am J Anat 1942;71: 371-415.

35 Waldo CM, Wislocki GB, Fawcett DW: Observations on the blood supply of growing antlers. Am J Anat 1949;84:27-61.

36 Rolf HJ, Enderle A: Hard fallow deer antler: a living bone till antler casting? Anat Rec 1999;255:68-77.

37 Kierdorf U, Schultz M, Fischer K: Effects of an antiandrogen treatment on the antler cycle of male fallow deer (Dama dama L.). J Exp Zool 1993;266:195-205.

38 Goss RJ: Inhibition of growth and shedding of antlers by sex hormones. Nature 1968;220: 83-85.

39 Goss RJ, Van Praagh A, Brewer P: The mechanism of antler casting in the fallow deer. J Exp Zool 1992;264:429-436.

40 Kierdorf U, Kierdorf H: Der Doppelkopf, ein für das Verständnis der Folgegeweih-Bildung aufschlussreiches Naturexperiment. Z Jagdwiss 1992;38:244-251.

41 Kierdorf U, Stoffels E, Stoffels D, Kierdorf H, Szuwart T, Clemen G: Histological studies of bone formation during pedicle restoration and early antler regeneration in roe deer and fallow deer. Anat Rec A 2003;273A:741-751.

-42 Li C, Suttie JM, Clark DE: Histological examination of antler regeneration in red deer (Cervus elaphus). Anat Rec A 2005;282A: 163-174.

43 Kierdorf H, Kierdorf U: State of determination of the antlerogenic tissues with special reference to double-head formation; in Brown RD (ed): The Biology of Deer. New York, Springer, 1992, pp 525-531.

44 Stoick-Cooper CL, Moon RT, Weidinger G: Advances in signaling in vertebrate regeneration as a prelude to regenerative medicine. Genes Dev 2007;21:1292-1315.

45 Goss RJ: Wound healing and antler regeneration; in Maibach HI, Rovee DT (eds): Epidermal Wound Healing. Chicago, Year Book Medical Publishers, 1972, pp 219-228.
46 Harty M, Neff AW, King MW, Mescher AL: Regeneration or scarring: an immunologic perspective. Dev Dyn 2003;226:268-279.

47 Goss RJ: Future directions in antler research. Anat Rec 1995;241:291-302.

48 Clark RAF: Wound repair. Overview and general considerations; in Clark RAF (ed): The Molecular and Cellular Biology of Wound Repair, ed 2. New York, Plenum Press, 1996, pp 3-50.

49 Ito M, Yang Z, Andl T, Cui C, Kim N, Millar SE, Cotsarelis G: Wnt-dependent de novo hair follicle regeneration in adult mouse skin after wounding. Nature 2007;447:316-320.

50 Ferguson MWJ, O'Kane S: Scar-free healing: from embryonic mechanisms to adult therapeutic intervention. Phil Trans R Soc Lond B 2004;359:839-850.

- 51 Eming SA, Hammerschmidt M, Krieg T, Roers A: Interrelation of immunity and tissue repair or regeneration. Semin Cell Dev Biol 2009;20:517-527.

52 Akimenko MA, Smith A: Paired fin repair and regeneration; in Hall BK (ed): Fins into Limbs: Evolution, Development, and Transformation. Chicago, University of Chicago Press, 2007, pp 152-162.

53 Mescher AL: The cellular basis of limb regeneration in urodeles. Int J Dev Biol 1996; 40:785-795.

54 Gyurján I, Molnár A, Borsy A, Stéger V, Hackler L, Zomborsky Z, Papp P, Duda E, Deák F, Lakatos P, Puskás LG, Orosz L: Gene expression dynamics in deer antler: mesenchymal differentiation towards chondrogenesis. Mol Genet Genomics 2007;277:221-235.

55 Li C, Yang F, Li G, Gao X, Xing X, Wei H, Deng X, Clark DE: Antler regeneration: a dependent process of stem tissue primed via interaction with its enveloping skin. J Exp Zool 2007;307A:95-105.

56 Kierdorf U, Kierdorf H, Schultz M: The macroscopic and microscopic structure of double-head antlers and pedicle bone of Cervidae (Mammalia, Artiodactyla). Ann Anat 1994;176:251-257.

57 Li C, Mackintosh CG, Martin SK, Clark DE: Identification of key tissue type for antler regeneration through pedicle periosteum deletion. Cell Tissue Res 2007;328:65-75.

-58 Kragl M, Knapp D, Nacu E, Khattak S, Maden M, Epperlein HH, Tanaka EM: Cells keep a memory of their tissue origin during axolotl limb regeneration. Nature 2009;460: 60-65.

59 Morrison JI, Lööf S, He P, Simon A: Salamander limb regeneration involves the activation of a multipotent skeletal muscle satellite cell population. J Cell Biol 2006;172: 433-440.

60 Echeverri K, Clarke JDW, Tanaka EM: In vivo imaging indicates muscle fiber dedifferentiation is a major contributor to the regenerating tail blastema. Dev Biol 2001;236:151164. 
-61 Gargioli C, Slack JWM: Cell lineage tracing during Xenopus tail regeneration. Development 2004;131:2669-2679.

62 Nechiporuk A, Keating MT: A proliferation gradient between proximal and $m s x b$-expressing distal blastema directs zebrafish fin regeneration. Development 2002;129:26072617.

63 Rolf HJ, Kierdorf U, Kierdorf H, Schulz J, Seymour N, Schliephake H, Napp J, Niebert S, Wölfel H, Wiese KG: Localization and characterization of STRO-1+ cells in the deer pedicle and regenerating antler. PLoS One 2008;3:e2064.

64 Mount JG, Muzylak M, Allen S, Okushima S, Althnaian T, McGonnell IM, Price JS: Antlers may regenerate from persistent neural crestlike stem cells; in Bartoš L, Dušek A, Kotrba R, Bartošová-Víchová J (eds): Advances in Deer Biology. Proc 5th Int Deer Biol Congress, Prague, 2006, p 161.

65 Price JS, Faucheux C: Exploring the molecular mechanisms of antler regeneration; in Sim JS, Sunwoo HH, Hudson RJ, Jeon BT (eds): Antler Science and Product Technology. Edmonton, ASPTRC, 2001, pp 53-67.
66 Banks WJ, Newbrey JW: Light microscopic studies of the ossification process in developing antlers; in Brown RD (ed): Antler Development in Cervidae. Kingsville, Caesar Kleberg Wildlife Research Institute, 1983, pp 231-260.

67 Colitti M, Allen SP, Price JS: Programmed cell death in the regenerating deer antler. J Anat 2005;207:339-351.

68 Kierdorf H, Kierdorf U, Szuwart T, Clemen G: A light microscopic study of primary antler development in fallow deer (Dama dama). Ann Anat 1995; 177:525-532.

69 Kierdorf U, Kierdorf H, Schultz M, Rolf HJ: Histological structure of antlers in castrated male fallow deer (Dama dama). Anat Rec A 2004;281A:1352-1362.

70 Mount JG, Muzylak M, Allen S, Althnaian T, McGonnell IM, Price JS: Evidence that the canonical Wnt signalling pathway regulates deer antler regeneration. Dev Dyn 2006;235: 1390-1399.

71 Allen SP, Maden M, Price JS: A role for retinoic acid in regulating the regeneration of deer antlers. Dev Biol 2002;251:409-423.

72 Kierdorf U, Bartoš L: Treatment of the growing pedicle with retinoic acid increased the size of first antlers in fallow deer (Dama dama L.). Comp Biochem Physiol C 1999; 124:7-9.

73 Kierdorf U, Kierdorf H: Effects of retinoic acid on pedicle and first antler growth in a fallow buck (Dama dama L.). Ann Anat 1998;180:373-375.
74 Suttie JM, Fennessy PF: Regrowth of amputated velvet antlers with and without innervation. J Exp Zool 1985;234:359-366.

75 Bubenik GA: The role of the nervous system in the growth of antlers; in Bubenik GA, Bubenik AB (eds): Horns, Pronghorns, and Antlers. New York, Springer, 1990, pp 339358.

76 Steinwedel M: Histologische und histogenetische Studien über das Geweih von Cervus elaphus, von Cervus capreolus und Rangifer tarandus; zugleich ein Beitrag zur Kenntnis des Verknöcherungs-Vorganges; doctoral thesis, Tierärztliche Hochschule Berlin, 1929, pp 1-81.

77 Kierdorf H, Kierdorf U: Knochengeschwulst an Damhirschstange. Z Jagdwiss 1985;31; 52-55.

78 Oviedo NJ, Beane WS: Regeneration: the origin of cancer or a possible cure? Semin Cell Dev Biol 2009;20:557-564.

79 Prehn RT: Regeneration versus neoplastic growth. Carcinogenesis 1997;18:1439-1444.

80 Goss RJ: Tumor-like growth of antlers in castrated fallow deer: an electron microscopic study. Scanning Microsc 1990;4:715-721. 